\title{
TOWARDS A NEW DEFINITION OF THE SECOND STAGE OF LABOUR
}

\section{Faisal Basama, MRCOG; Katherine Granger, MRCOG}

\section{RATIONALE}

The idea of this audit stemmed from the critical incident reporting in the maternity unit, where the majority of the incident reports were of prolonged second stage of labour. There were concerns that a prolonged or inappropriately managed second stage may have longterm effects on the maternal and fetal health.

The lack of definition or management guidelines for the second stage of labour may have contributed to its prolongation and to the increased rates of interventions. There was recognition that this area of intrapartum care, especially when inappropriately managed, may perhaps lead to litigation.

\section{INTRODUCTION}

The second stage of labour is defined as: the part of labour that starts with the full dilatation of the cervix and is terminated when the child is fully expelled and its duration should continue no longer than one hour in multiparae and two hours in primiparae. Many factors have been blamed for the prolongation of the second stage; fetal malposition at full dilatation, (1) parity, oxytocic augmentation, and epidural analgesia. ${ }^{(1,2)}$ However, the exact mechanisms of their influences still lack understanding.

Childbirth in general, and prolonged second stage of labour in particular, increases maternal morbidity; perineal trauma, third degree anal sphincter tears, and postpartum haemorrhage..$^{(3,4)}$ Prolonged second stage of labour is associated with increased rates of operative deliveries. ${ }^{(3)}$ Interventions to shorten the second stage of labour (episiotomies and assisted vaginal deliveries) may cause anal sphincter damage. ${ }^{(6)}$ The damage, usually, involves all the elements of the pelvic floor; the perineal body, the muscles, the nerves, and the collagen and connective tissues.

\section{METHODS}

Site

The audit was conducted at the Royal Lancaster Infirmary, a district general teaching hospital in the north-west of England with a delivery rate of approximately 2,500 births per annum.

\section{Subjects}

The notes of 107 women who had a prolonged second stage of labour were reviewed for the audit.

\section{Time}

The audit covered the period from 01 January to 31 December, 2004.

\section{Data collection}

This was a retrospective audit. The computer programme Protos was used to identify those women who had a prolonged second stage of labour in the specified period 336 women were identified (approximately $13.44 \%$ of all deliveries). There were 207 primigravidae and 129 multiparae. Following consultation with the audit team, it was agreed that 100 casenotes would be sufficient for this audit; hence, only 107 women (4.28\% of all deliveries) were randomly selected for the audit. The data was collected through a designed proforma. The identified women's casenotes were extracted and evaluated.

\section{The audit standards}

The unit's practice is based on the traditional standards for the management of the second stage of labour:

- the duration of the second stage of labour should not last for more than one hour for a multiparous woman

- the duration of the second stage of labour should not last for more than two hours for a nulliparous woman

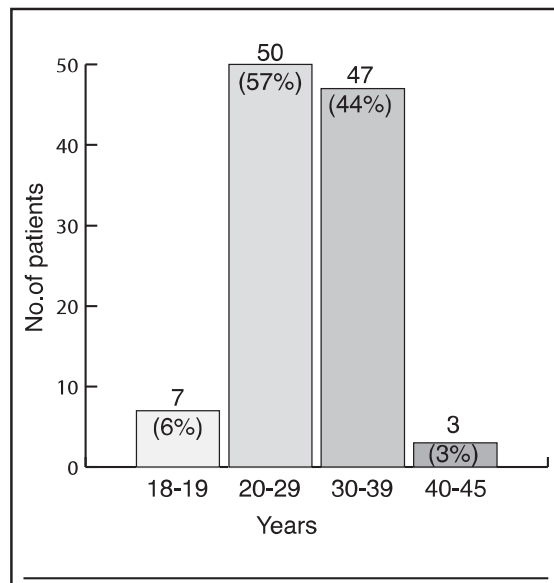

Figure 1 Age distribution $(n=107)$
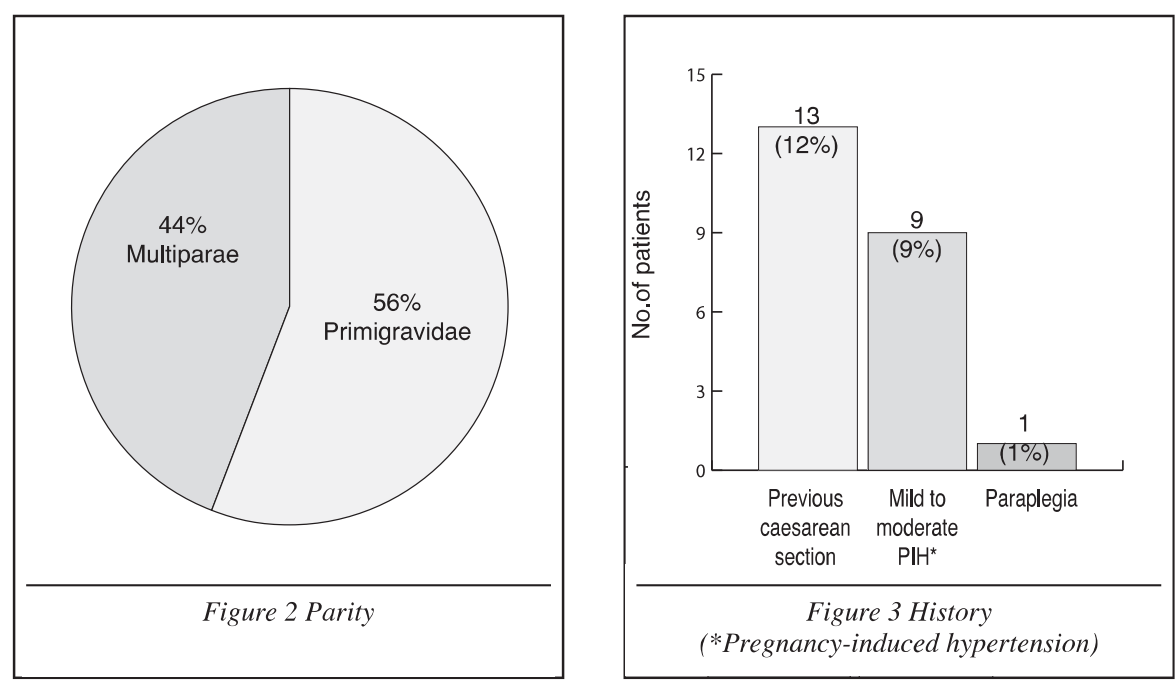

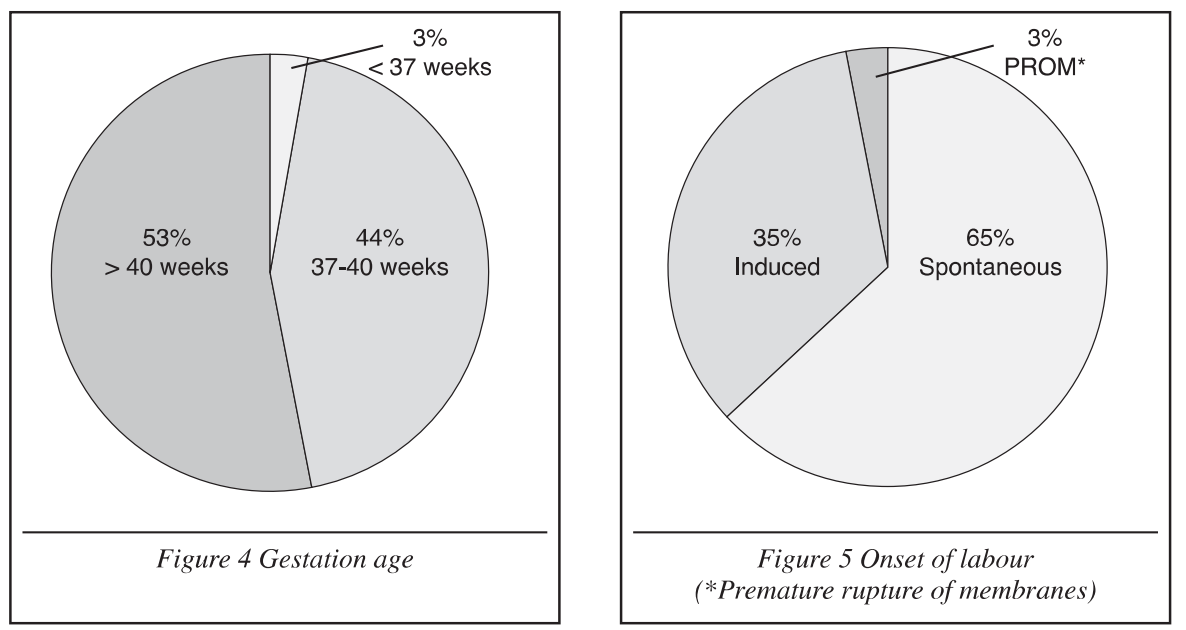

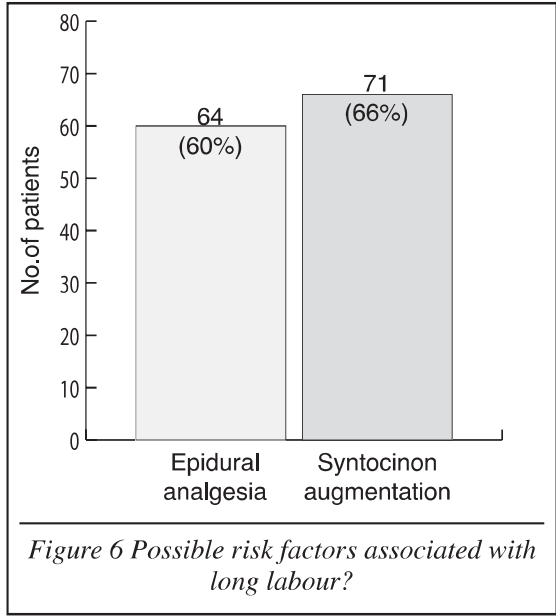

\section{RESULTS}

Figures 1 to 9 show the various results of the audit. There was a median age of 29 years. Of those requiring syntocinon augmentation (see figure 6), $85 \%$ were primiparae and $43 \%$ multiparae. Of those who had epidural analgesia (see figure $6)$, the liquor was stained with meconium in $15(14 \%)$ of all labours and in $11(15 \%)$ of the augmented ones. All the women had fetal monitoring. Nineteen women (20\%) had unsatisfactory fetal CTG and four had meconium-stained liquor as well; one of these babies was admitted to the special care baby unit (SCBU).

The duration of the second stage of labour was 02:01 to 04:56 hours for primiparae with a median of 03:01 hours, and 01:01 to $04: 45$ hours for mutliparas with a median of $01: 32$ hours. Eight of the women with meconium-stained liquor $(60 \%)$ had instrumental delivery and two (15\%) had caesarean section. The prolongation of the second stage was not explained in $69(64 \%)$ of the women, while a reason was given in 38 (36\%); malposition in 16 women, maternal exhaustion in 11, inadequate or incordinate uterine activity in ten, and one case of cephalo-pelvic disproportion.

Four (4\%) of all babies were admitted to the SCBU for a short period of time. Twenty-nine women (27\%) had blood loss of more than 500 millilitres. The loss was between 500 to 1,000 millilitres in $23 \%$, and $>1,000$ to 1,500 millilitres in $2 \%$. One woman lost $>2,000$ to 2,500 millilitres and another woman lost $>2,500$ to 3,000 millilitres. Sixty-eight $(63 \%)$ women had a second degree perineal tear, $3 \%$ had a third degree, and $2 \%$ had a fourth degree tear. Three percent of the women had postpartum voiding difficulties.

\section{DISCUSSION}

The current climate of the NHS favours evidence-based practice in all aspects of patients' care; including obstetrics and intrapartum care. The lack of definition and management guidelines for the second stage of labour are, probably, contributing to the increased incidence of prolonged second stage and operative deliveries. The traditional definition of the second stage of labour, which is based on Friedman's Partogram, may not truly represent the different dynamics of labour in different women's populations. There is no evidence to support the allocation of time for the second stage of labour or its management; hence, both may lead to confusion and dispute with an increased potential for conflict between the different health professionals who contribute to the care of the women in labour. Our reference here is the obstetrics/midwifery boundary, which is sensitive and represents the transition from labour as a normal physiological process to an abnormal one, which not infrequently necessitates medical intervention.

There seems to be a need to review the different mechanisms of the second stage of labour. Such a review may ultimately involve a redefinition of the duration and management of the second stage of labour. Fifty years after the original description of the second stage by Friedman it is claimed that the average length of labour for primiparae and
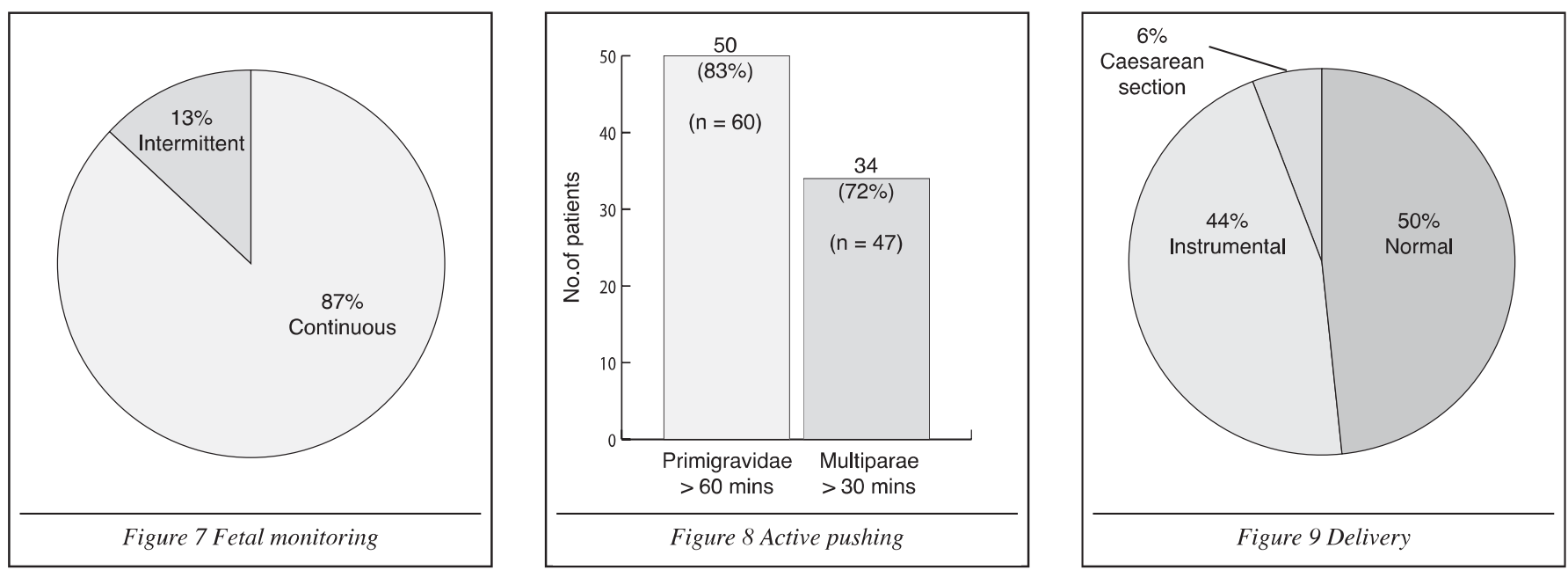


\section{PEER REVIEWS}

The editor invited senior colleagues in the Trust to comment on this audit and is grateful to them for their insight. Sharon Hayes is Matron, Central Delivery Suite, Royal Lancaster Infirmary.

The management of prolonged second stage remains problematic for both obstetrician and midwife. There are a number of factors which have to be considered although like the authors I believe that not one of these factors can be singled out to be determined as causative

As the terms of reference of the audit did not differentiate between passive and active second stage of labour, I feel that a re-audit making this differentiation may be more enlightening as it is now accepted evidence-based practice to allow one hour for descent of the presenting part prior to commencing active second stage of labour, with a direct consequence that the picture may not be as negative as depicted.

Since the audit, in 2006, the Maternity Risk Steering Group has re-evaluated the trigger for completion of a clinical risk form which clearly demarcates between passive and second stage of labour. This was endorsed by the publication of NICE Clinical Guideline No. 55 Intrapartum Care, in September 2007, which fully supports the redefinition.

\section{Mandy Baldam is a Consultant Anaesthetist, Royal Lancaster Infirmary. She has specific responsibility for leading the obstetric anaesthesia service.}

One hundred and seven patients, although a small group, does at least give a picture of what was happening in this time period.

\section{A couple of comments:}

- this is a heterogenous group of patients which has a bias towards a higher epidural rate of $60 \%$ compared to the overall rate of around $25 \%$

- in addition, they also display a higher than average rate of augmentation of labour and as noted in the discussion it is impossible to separate or link these two factors without more information

The high incidence of induction and augmentation of labour may be characteristic of a group with a higher tendency to need interventions. For valid reasons women may be more likely to request or be advised to have an epidural. It is of course difficult to conduct valuable studies comparing epidural vs. no epidural and the evidence is conflicting. I agree that future audit could look at different populations and we may get more useful findings

At the Royal Lancaster Infirmary, we use a low-dose regimen for epidural top-ups; $15 \mathrm{ml}$ of $0.1 \%$ bupivacaine and fentanyl $2 \mathrm{mcg} / \mathrm{ml}$. This method of delivering analgesia was introduced by my colleague Sue Harding as long ago as 1996 and her experience, professional and personal, was reported in the Journal in 1998. Her initial impression that this was a valuable technique was confirmed by the COMET trial, reported in the Lancet in 2001, which showed that lowdose epidurals result in better rates of normal vaginal delivery than traditional $0.25 \%$ bupivacaine doses - $43 \%$ compared with $35 \%$.

multiparae today is similar to the average length of labour then, but there is now a wider range of normal.(7) A prolonged second stage, of up to eight hours in primiparae and four and a half hours in multiparae, is claimed not to be associated with adverse effects to mother or infant, justifying a revision of Friedman's Labour Curve to meet the needs of current women's populations, technological advances, and nursing responsibilities. The conclusion is that the duration of a satisfactorily progressing labour should be allowed to progress past the rigid two-hour time limit.

Many would agree that the first hurdle in the management of the second stage of labour is the diagnosis of its onset, which is inaccurate and difficult to ascertain. No practitioner can be certain that full dilatation has occurred, precisely, at the time of the examination. That can only be ascertained through continuous or very frequent assessment of the cervix, which is clinically unacceptable.

Any discussion of the management of labour is incomplete if pain management is not included. In modern obstetrics, epidural analgesia has become an essential part of the service; $60 \%$ of the women in this audit had epidural analgesia. The evidence for and against this type of analgesia is conflicting. It has been claimed that epidural analgesia may both prolong labour and increase non-spontaneous deliveries, ${ }^{(8,9)}$ but it has also been shown to be effective in reducing pain during labour and may be beneficial for both the mother and fetus. ${ }^{(10,11,12,13)}$ A recent meta-analysis demonstrated that primiparous women with epidurals were likely to have fewer 
rotational or mid-cavity operative interventions when pushing was delayed for one to two hours or until they had a strong urge to push. ${ }^{(14)}$ There is insufficient evidence to support the hypothesis that discontinuing epidural analgesia reduces the incidence of operative vaginal delivery; however, that may increase women's pain. It seems that there is no difference between the rates of operative vaginal delivery for combined spinal-epidural and epidural techniques..$^{(15)}$

The use of oxytocin in the second stage of labour in primiparous women with epidural anaesthesia can reduce the need for non-rotational forceps delivery. ${ }^{(16)}$ We are aware that the augmentation of the labour with syntocinon in the multiparous is not uncommon. However, we view the practice with great suspicion, especially when employed in the prolonged second stage of labour. Such an intervention is highly contentious and requires experience, high levels of caution, and close monitoring.

The effect of active pushing on the duration of the second stage and the outcome of labour is not fully investigated, especially in women who received epidural analgesia. There is evidence that active pushing in the second stage is associated with significantly greater risk for denervation injury compared with passive and lesser amount of pushing, ${ }^{(17)}$ and that damage to the pelvic floor muscles, nerves, and connective tissue may lead to stress urinary incontinence in the future. On the other hand, spontaneous pushing during the second stage of labour, compared with directed pushing, is more likely to result in intact perineum, fewer episiotomies or second or third degree lacerations. ${ }^{(18)}$ It has also been observed that delayed pushing prolonged labour, but did not result in significantly higher rates of altered continence or anal sphincter injury, when compared with immediate pushing. ${ }^{(19)}$

This audit has shown the duration of the second stage of labour was nearly five hours in both primiparae and multiparae, which is, in terms of traditional definition, definitely prolonged. However, it has to be recognised that this audit does not distinguish between passive as opposed to active second of labour. There might be a case for the prolongation of the second stage of labour, if the maternal and fetal surveillance are normal. Recently, an attractive reconceptualisation of the second stage of labour has been proposed. ${ }^{(20)}$ The proposal recognises two phases, an early phase of descent and a later phase of active pushing, and allows for a longer early phase and a shorter active phase, when the woman is encouraged to push only when the obstetric conditions are optimal. The longterm effects of such policies on the pelvic floor muscles need further investigation. However, the proposal leaves the door open for different interpretations of the term 'optimal obstetric conditions' and there is a legitimate concern that this argument could be the same gate that leads to increased interventions and maternal morbidity.

Earlier studies have not observed any detrimental effect of prolonged second stage of labour on the neonatal outcome..$^{(2,3,5)}$ The possible explanation for this observation is probably a lowered threshold for intervention to prevent fetal hypoxia that is probably achieved on the expense of an increased maternal morbidity.

We recognised that the population of the audit is not homogenous; however, it fairly represents our women's population and reflects our practice. We also recognised that the women who had their labours induced or augmented may be over-represented. We thought that women who required induction of labour for post dates or augmentation may represent a complex population who have complicated labours due to problems; such as deflexed head and relative cephalo-pelvic disproportion. Such a population may have inherited tendencies towards abnormal labour, incordinateuterine contractions, and high possibility of interventions such as oxytocic augmentation and epidural analgesia.

Perhaps, it would have been more meaningful if these women were assessed as different populations of primiparae, multiparae, spontaneous labour, induced labour, trial of scar, and those with or without epidural analgesia; which could be an idea for a future audit project.

One possible preventive strategy is a structured training programme for the pelvic floor muscles, to reduce the incidence of prolonged second stage of labour deserves further exploration. The intervention is physiological, practical, and cheaper than most of the remedies for maternal and fetal morbidities associated with prolonged second stage of labour.

We noticed that there was no explanation for the prolongation of the second stage of labour in $64 \%$ of the women in this audit. There is a need to emphasise the importance of assessment and documentation of the fetal position and station in vaginal assessments. It is suspected that this figure will be significantly lower in future audits, especially if senior and middle-grade clinicians would put extra effort to provide a logical explanation for some of the abnormal labours.

Malposition of the fetal head, cephalo-pelvic disproportion, parity, maternal exhaustion, and inadequate or incordinate uterine activity were among the parameters recognised in this audit, and by other observers, ${ }^{(1)}$ to be frequently associated with prolonged second stage of labour and increased maternal morbidity. However, the association with parameters such as oxytocics augmentation and epidural analgesia ${ }^{(1,2)}$ is better viewed as an intervention to correct an abnormal labour mechanism rather than a cause.

Finally, it is worth mentioning that the correct interpretation and usage of the partogram should lead to fewer operative births and less use of oxytocin.

\section{CONCLUSION}

The population of the audit was heterogeneous and the number of the cases was small, therefore the results of this audit should be carefully interpreted.

There was failure to explain the prolongation of the second stage of labour in the majority of the cases. There was a high rate of labour induction, syntocinon augmentation, and assisted vaginal deliveries; however, the caesarean section rate was not high. There was a high incidence of perineal damage; however, the incidence of immediate, postpartum voiding difficulties was low.

\section{RECOMMENDATIONS}

A re-audit that adopts a systemic approach in the evaluation of prolonged second stage is needed. The cohort should include all the women with prolonged second stage, in order 
to give true representation of the population. The women's population should be categorised as primiparae, multiparae, spontaneous labour, induced labour, trial of scar, and those with or without epidural analgesia.

There is a need for evidence-based guidelines or consensus on the management of the second stage of labour, especially its duration and active pushing.

The potentials of preventive strategies, such as a structured training programme for the pelvic floor muscles, which may reduce the incidence of prolonged second stage of labour, deserves exploration.

\section{REFERENCES}

1. Schiessl B, Janni W, Jundt K, Rammel G, Peschers U, Kainer F. Obstetrical parameters influencing the duration of the second stage of labor. Eur J Obstet Gynecol Reprod Biol 2005;118(1):17-20

2. O'Connell MP, Hussain J, Maclennan FA, Lindow SW. Factors associated with a prolonged second state of labour - a case-controlled study of 364 nulliparous labours. J Obstet Gynaecol 2003;23(3):255-7

3. Cheng YW, Hopkins LM, Caughey AB. How long is too long: Does a prolonged second stage of labour in nulliparous women affect maternal and neonatal outcome? Am J Obstet Gynecol 2004;191(3):933-8

4. Sultan AH, Kamm MA, Hudson CN. Pudendal nerve damage during labour: prospective study before and after childbirth. Br J Obstet Gynaecol 1994;101(1):22-8

5. Janni W, Schiessl B, Peschers U, et al. The prognostic impact of a prolonged second stage of labor on maternal and fetal outcome. Acta Obstet Gynecol Scand 2002;81(3):214-21

6. Eason E, Labrecque M, Marcoux S, Mondor M. Anal incontinence after birth. Can Med Assoc J 2002;166(3):326-30

7. Cesario SK. Reevaluation of Friedman's Labor Curve: a pilot study. J Obstet Gynecol Neonatal Nurs 2004;33(6):713-22

8. Leong EW, Sivanesaratnam V, Oh LL, Chan YK. Epidural analgesia in primigravidae in spontaneous labour at term: a prospective study. J Obstet Gynaecol Res 2000;26(4):271-5

9.Zimmer EZ, Jakobi P, Itskovitz-Eldor J, et al. Adverse effects of epidural analgesia in labor. Eur $\mathrm{J}$ Obstet Gynecol Reprod Biol 2000;89(2):153-7
10. Anim-Somuah M, Smyth R, Howell C. Epidural versus non-epidural in labour. Cochrane Database Syst Rev 2005;(4):CD000331

11. Boder-Adler B, Bodner K, Kimberger O, et al. The effect of epidural analgesia on obstetric lacerations and neonatal outcome during spontaneous vaginal delivery. Arch Gynecol Obstet 2003;267(3):130-3

12. Clark A, Carr D, Loyd G, Cook V, Spinnato J. The influence of epidural analgesia on cesarean delivery rates: a randomized, prospective clinical trial. Am J Obstet Gynecol 1998;179(6 Pt 1):1527-33

13. Halpern SH, Leighton BL, Ohlsson A, Barrett JF, Rice A. Effect of epidural vs parenteral opioid analgesia on the progress of labor: a meta-analysis. JAMA 1998;280(24):2105-10

14. Roberts CL, Torvaldsen S, Cameron CA, Olive E. Delayed versus early pushing in women with epidural analgesia: a systematic review and meta-analysis. BJOG 2004;111(12):1333-40

15. Hughes D, Simmons SW, Brown J, Cyna AM. Combined spinal-epidural versus epidural analgesia in labour. Cochrane Database Syst Rev 2003;(4):CD003401. [DOI:10.1002/14651858]

16. Saunders NJ, Spiby H, Gilbert L, et al. Oxytocin infusion during second stage of labour in primiparous women using epidural analgesia: a randomized double-blind placebo-controlled trial. Br Med J 1989;299:1423-6

17. Allen RE, Hasker GL, Smith AR, Warrell DW. Pelvic floor damage and childbirth: a neurophysiological study. Br J Obstet Gynaecol 1990;97(9):770-9

18. Sampselle C, Hines S. Spontaneous pushing during birthrelationship to perineal outcomes. J Nurse Midwifery 1998;44(1):36-9

19. Fitzpatrick M, Harkin R, McQuillan K, O'Brien C, O'Connell PR, O'Herlihy C. A randomised clinical trial comparing the effects of delayed versus immediate pushing with epidural analgesia on the mode of delivery and faecal continence. BJOG 2002;109(12):1359-65

20. Roberts JE. A new understanding of the second stage of labor: implications for nursing care. J Obstet Gynecol Neonatal Nurs 2003;32(6):794-801 